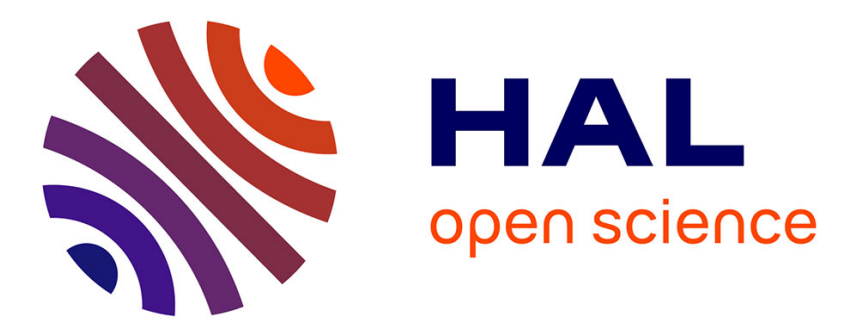

\title{
Preliminary measurement of the spherical proportional counter prototype
}

Zhi-Min Wang, Charling Tao, Chang-Jiang Dai, Hai-Qiong Zhang, Peng Zhang, Ning Zhou, Yi Tao, Chen-Yang Tang, Rui-Guang Wang, Chang-Gen Yang, et al.

\section{To cite this version:}

Zhi-Min Wang, Charling Tao, Chang-Jiang Dai, Hai-Qiong Zhang, Peng Zhang, et al.. Preliminary measurement of the spherical proportional counter prototype. Radiat.Detect.Tech.Meth., 2018, 2 (1), pp.18. 10.1007/s41605-018-0045-3 . hal-02017068

\section{HAL Id: hal-02017068 https://hal.science/hal-02017068}

Submitted on 4 Apr 2019

HAL is a multi-disciplinary open access archive for the deposit and dissemination of scientific research documents, whether they are published or not. The documents may come from teaching and research institutions in France or abroad, or from public or private research centers.
L'archive ouverte pluridisciplinaire HAL, est destinée au dépôt et à la diffusion de documents scientifiques de niveau recherche, publiés ou non, émanant des établissements d'enseignement et de recherche français ou étrangers, des laboratoires publics ou privés. 


\title{
Preliminary Measurement of the Spherical Proportional Counter Prototype
}

\author{
Zhi-Min Wang ${ }^{1,2 ; 1) *}$, Charling Tao ${ }^{2,3 ; 2) \dagger}$, Chang-Jiang Dai ${ }^{1,2}$, \\ Hai-Qiong Zhang ${ }^{1,4}$, Peng Zhang ${ }^{1}$, Ning Zhou ${ }^{2}$, Yi Tao ${ }^{2}$, \\ Chen-yang Tang ${ }^{2}$, Rui-Guang Wang ${ }^{1}$, Chang-Gen Yang ${ }^{1}$, \\ Ruo-qing Liu ${ }^{2}$ \\ ${ }^{1}$ Institute of High Energy Physics, Chinese Academy of Sciences, Beijing 100049, China \\ ${ }^{2}$ Tsinghua University, Beijing 100084, China \\ ${ }^{3}$ Marseille Particle Physics Center (CPPM), Maseilles 13233, France \\ ${ }^{4}$ University of Chinese Academy of Science, Beijing, China
}

\begin{abstract}
A novel large volume spherical proportional counter aims at relatively large target masses with low threshold, low background, possible particle identification, with an extremely simple and robust operation. In the future, it is possible to detect neutrino-nucleus interaction, supernova detection, low energy neutrino oscillations and study of non-standard properties of the neutrino, dark matter, axion, ... In this paper, we will present some progress on the study of such a kind of detector, with a prototype in Beijing for $\alpha$, neutron measurement and long term running stability.
\end{abstract}

Keywords: keywords: Gas detector, SPC, Alpha, neutron, PSD

\section{Introduction}

The study of rare event physics, such as neutrino [1-3], WIMP [4-6], axion $[7], \ldots$, requires detectors with large target mass, low threshold and low background. The Spherical Proportional Counter (SPC) is a novel concep$\mathrm{t}$ [8-10] with very promising features, among which is the possibility of easily instrumenting large target mass with very low energy threshold and particle

\footnotetext{
*wangzhm@ihep.ac.cn

†tao@cppm.in2p3.fr
} 
identification. This could open a way of detecting the tiny (few hundreds of $\mathrm{eV}$ ) nuclear recoils produced by neutrino-nucleus coherent interaction, which, although a process foreseen in the "Standard Model" of particle physics, has never been within reach of current detectors sensitivities. A first prototype had been built in Saclay to demonstrate the concept [11, 12]. Preliminary tests have proven issues like robustness, gain and stability of operation [13]. In spite of the simplicity of the operation, some spatial resolution is achieved in the radial coordinate by inspecting the time pattern of the charge pulse detected at the center of the sphere. One SPC prototype was setup in Beijing for studying the detector performance, in a collaboration between Saclay, CPPM/IN2P3, Tsinghua and IHEP.

In this work, we will present the first results from Beijing SPC prototype on detector response.

\section{Spherical Proportional Counter (SPC)}

The SPC is made up by 2 concentric spheres (Fig. 1, [14]): the external one connected at ground and the inner one at high potential as a collection pole. The external sphere also runs as a vessel that tightly encloses the target gas inside as the drift volume. The ionization charges produced in the interaction, drift towards the center, are multiplied in the strong electric field near the center field and readout by the electronics. In the design, the amplification structure is just a small spherical electrode, around which the avalanche is produced, and read as a single electronics channel.

The spherical symmetry and small dimension of the central anode means that the detector capacity is very low, allowing for extremely low levels of electronic noise. Its temporal extension is determined by the longitudinal diffusion of the ionization cloud and therefore by the distance drifted. The $\frac{1}{r^{2}}$ dependence of the electric field enhances this effect when compared to plane or cylindrical TPCs. Preliminary tests with a prototype have easily achieved thresholds of $200 \mathrm{eV}$. The natural radial focusing of the spherical geometry allows the collection and amplification of the deposited charges using a single electronic channel to read out a large volume. In principle, the simplicity of its design should also allow an easy optimization from the point of view of radio purity. The spherical symmetry minimizes the external surfaces per unit of detector volume, as well as the thickness of material needed to hold the gas, therefore allowing a lower background per unit volume due to external surface or material contaminations. 


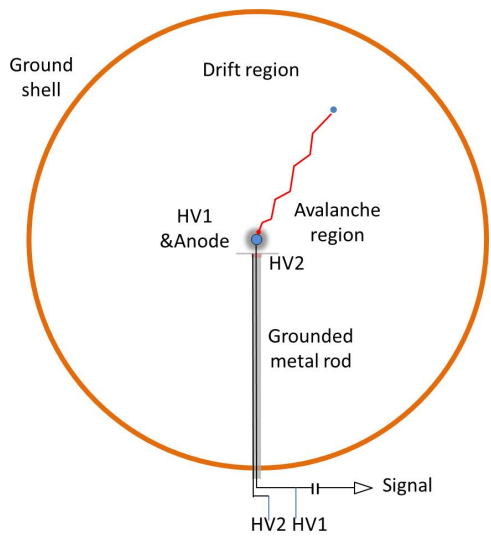

Figure 1: Schematic view of the spherical proportional counter and its detection principle.

The Beijing prototype detector (Fig. 2) consists of a large spherical copper vessel, $1.3 \mathrm{~m}$ in diameter and $6 \mathrm{~mm}$ thick. The spherical vessel is pumped (down to $10^{-5} \mathrm{~Pa}$ ) and then filled with a gas mixture at a pressure 0.05 to $0.2 \mathrm{~atm}$ for our studies, but high pressures of several atmospheres can also be used. A small copper ball, $14 \mathrm{~mm}$ in diameter, is fixed in the center of the spherical vessel by a copper rod and an umbrella in order to make the electronic field more uniform, acts as an electrode with positive high voltage and as a proportional amplification counter.

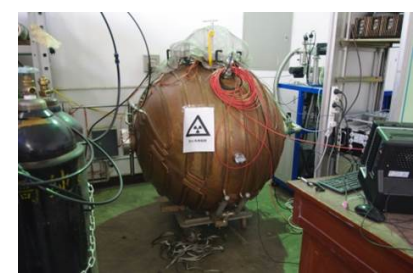

Figure 2: The real prototype detector setup at IHEP.

The electric field varies inversely to the distance squared $\left(\frac{1}{r^{2}}\right)$ and it is highly inhomogeneous along the radius. The difference in the intensity of the electric field, from the outer to the inner radius, divides the detector volume into two regions (Fig. 1): the drift and the amplification region. Primary ionization electrons in the drift region drift towards the anode (the drift time varies from $\mu$ s to $\mathrm{ms}$ depending on the gas mixture and pressure). When these electrons reach a distance of a few $\mathrm{mm}$ from the anode, the avalanche 
starts due to the intense electric field. The induced signal passes through a charge sensitive preamplifier, is read-out by an Analog to Digital Converter (ADC) and data is stored in a computer memory. The pulse shape of the signal depends on the charge spatial density distribution and the distance of the interaction from the anode. In principle, its main advantages of this detector, for low energy recoil detection (either neutrino or WIMP induced recoils), are the simple design, the large volumes achievable, the energy resolution in the low energy region and the low electronic noise provided by the low capacitance (even for large detector sizes). The low energy threshold of the detector is limited only by the mean ionization energy of the gas mixture. The detection range varies from a few $\mathrm{eV}$ to tenths of $\mathrm{MeV}$, depending on the amplification field, allowing detection of low energy gammas, electrons, $\alpha$ particles and heavy ions. Lastly, the fiducialization capability of the detector, allows us to distinguish point like energy depositions (low energy gammas, low energy electrons, heavy ions), from spatially extended depositions (muons), through pulse shape analysis.

\section{Detector Response}

Normally the SPC filled with designed gas mixture and pressure for specified detection goal. As a first test of the prototype, we select $A r(98 \%)+$ $\mathrm{CH}_{4}(2 \%)$ as the target with $5000 \mathrm{~Pa}$ or $20000 \mathrm{~Pa}$. In order to understand the basic features of the detector for neutron measurement, firstly we use $\alpha$ from thoron of ${ }^{232} \mathrm{Th}$ decay chain as the source to calibrate the detector response. As shown in Fig. 1, the signal of the detector is de-coupled firstly from the HV and amplified by a charge sensitive amplifier, then recorded to computer by a $2 \mathrm{MHz}$ sampling $\mathrm{ADC}$ in $2 \mathrm{~ms}$ window per trigger, where the trigger is generated by amplitude discrimination online. All the following analysis is based on the recorded waveform data (Fig. 3) with amplitude, charge and rise time.

\subsection{Internal Alpha}

In contrast to normal radon $\left({ }^{222} R n\right)$ from the Uranium chain, Thoron $\left({ }^{220} \mathrm{Rn}\right)$ from the Thorium chain, does not leave long lived radioactive deposits left in the detectors, which makes it an ideal $\alpha$ source for tests in detectors of rare events. Only ${ }^{212} \mathrm{~Pb}$ will remain with 11 hours half-life. The generated daughter isotopes provide mono-energetic $\alpha \mathrm{s}: \quad 6.2 \mathrm{MeV}$ from 


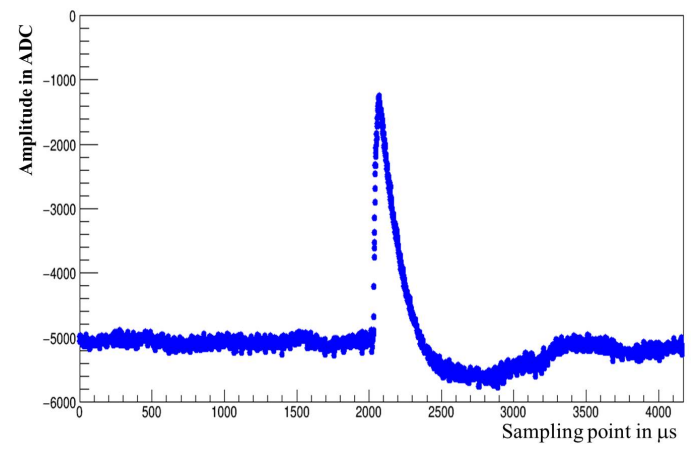

Figure 3: Sampled waveform: $\mathrm{X}$ axis is the sampling time in $\mu$ s and $\mathrm{Y}$ axis is the pulse amplitude in ADC.

${ }^{212} \mathrm{Bi}, 8.9 \mathrm{MeV}$ from ${ }^{212} \mathrm{Po}$, and the combination energy ( $11.4 \mathrm{MeV}$ ) of Beta ( $2.5 \mathrm{MeV}$ ) from ${ }^{212} \mathrm{Bi}$ and $\alpha$ from ${ }^{212} \mathrm{Po}$.

The detector is configured with $20 \mathrm{kPa}$ and $\mathrm{HV} 1 / \mathrm{HV} 2=3450 \mathrm{~V} / 800 \mathrm{~V}$ for possible $\alpha$ scale, with set threshold at 1500 ADC. The data is recorded a few hours after the gas is filled with ${ }^{220} R n$.

The spectra of pulse rise time and amplitude (energy) from background (without source) and source are compared as shown in Fig. 4, where the background is in blue, the source is in red and the pure source with background subtracted is in black. The source peaks on rise time and amplitude spectrum are clear. The cosmic ray, which is the main source of the background spectrum, has slower rise time and smaller amplitude compared to the internal $\alpha$. In the 2D graph of rise time Vs. amplitude with the source as in Fig. 5, the structure is more clear where it is possible to get better energy measurement with rise time cuts as shown in Fig. 6. From the Fig. 6 we can obtain the $\alpha$ energies as listed in Tab. 1. We also find a sharp structure around 6000 AD$\mathrm{C}$ on amplitude spectrum, which is consistent with electron assumption. It shows very promising feature for $\alpha$ measurement and alpha/electron separation. It is also possible to improve the energy resolution with specified gas mixture $\left(\mathrm{Ar}\right.$ and $\left.\mathrm{CH}_{4}\right)$ and pressure, suitable voltage potential of anode and umbrella.

\subsection{Neutron}

According to [15], a SPC filled with $\mathrm{N}_{2}$ and $\mathrm{Ar}+\mathrm{CH}_{4}$ mixture could have good neutron detection capability. In order to test its performance, we configured the prototype with $\mathrm{Ar}(89 \%)+\mathrm{N}_{2}(6 \%)+\mathrm{CH}_{4}(5 \%)$ and HV1/HV2 

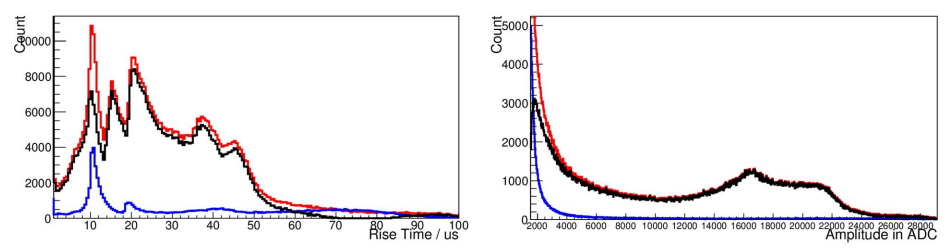

Figure 4: Left panel is the rise time distribution of signal; right panel is the amplitude spectra (Blue: background; red with $\alpha$ source; black: pure source with background subtracted).

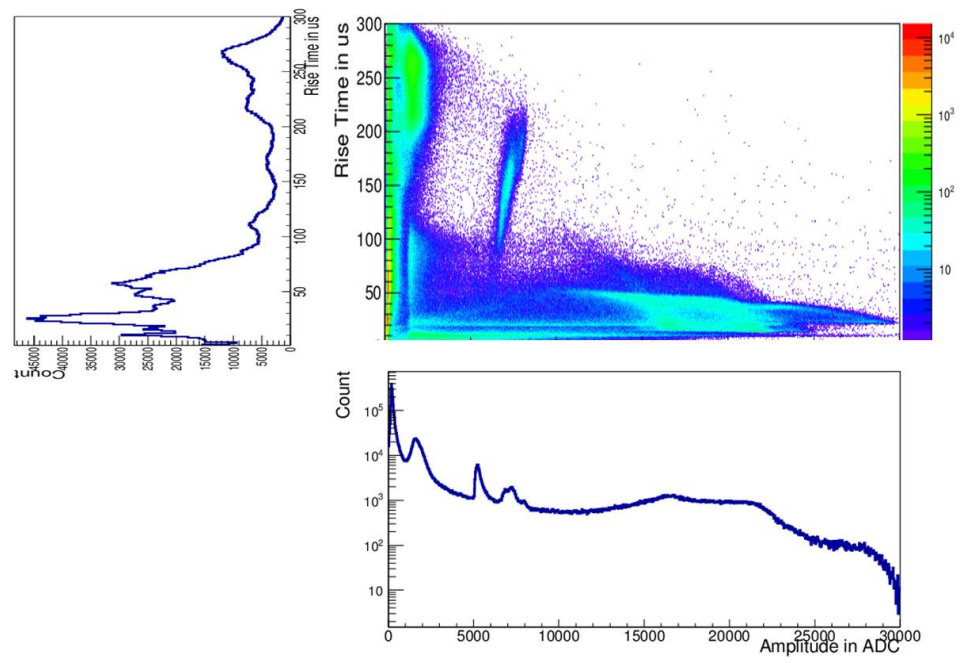

Figure 5: The 2D graph of pulse rise time and amplitude with the $\alpha$ source.

$=3600 \mathrm{~V} / 180 \mathrm{~V}$, with a threshold of $900 \mathrm{ADC}$ and $15 \mathrm{kPa}$. Here the calibration source is ${ }^{252} \mathrm{Cf}$.

From Fig. 7, the spectra comparison of background (Blue), source (Red) and pure source after background subtracted (black), the rise time of neutron events is very fast, where just selects the rise time less than 12us without considering the efficiency, and we get a peak in the energy spectrum as shown Fig. 8, which corresponds to thermal neutron captures. We observed that using nitrogen, with a larger target mass by higher pressure, is indeed an alternative to Helium3 for neutron detection. 


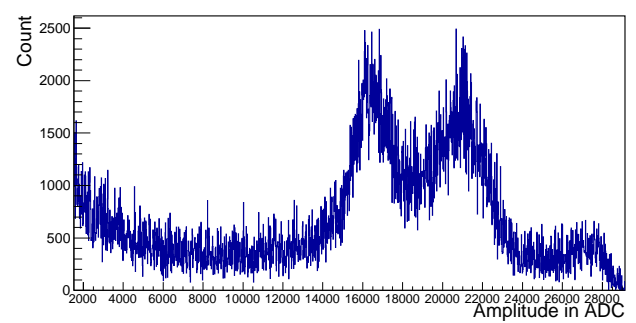

Figure 6: Amplitude spectrum with rise time cut $[25,30] \mu$ s for $\alpha$.

Table 1: $\alpha$ peaks and energy.

$\begin{array}{cc}\text { Energy }(\mathrm{MeV}) & \text { Amplitude }(A D C) \\ 6.2 & 16500 \\ 6.8 & 21000 \\ 8.9 & 26800\end{array}$

\subsection{X-ray}

In principle, the SPC detector is also a good instrument for low energy $\mathrm{X}$-ray with good resolution with lower pressure as tested by [8-10]. Here the detector is running at a pressure of $15 \mathrm{kPa}$ with $\mathrm{HV} 1 / \mathrm{HV} 2=4500 \mathrm{~V} / 850 \mathrm{~V}$, and $60 \mathrm{keV}$ from ${ }^{241} \mathrm{Am}$ with threshold $500 \mathrm{ADC}$.

The $60 \mathrm{keV}$ signal is not so clear from the rise time spectrum because of most the background also X-ray or Gamma as shown in Fig. 9. The deposited peak is located around 5000 ADC as shown in Fig. 10. The observed resolution is not so good where the reason still under investigation for out gasing, gas purity, bad central anode etc.
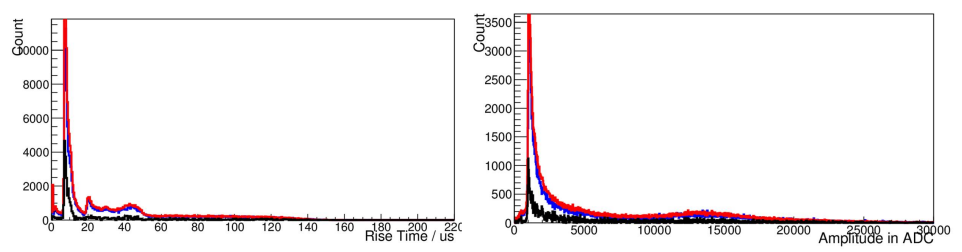

Figure 7: Left panel is the rise time distribution of the signal; right panel is the amplitude spectra (Blue: background; red with neutron source; black: pure source with background subtracte). 


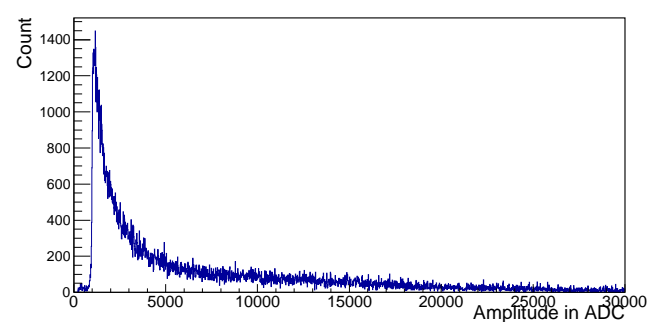

Figure 8: Amplitude spectrum after rise time cut $[6,12] \mu$ s for neutron.
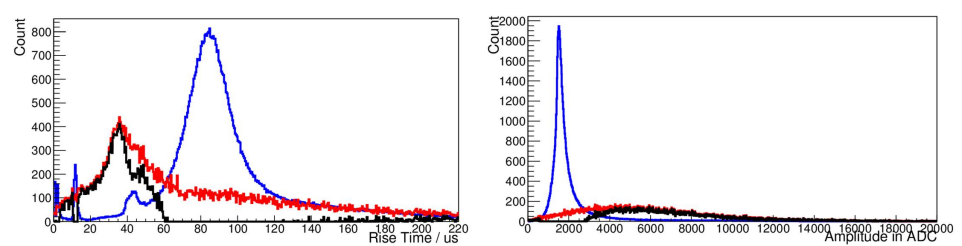

Figure 9: Left panel is the rise time distribution of the signal; right panel is the amplitude spectra (Blue: background; red with $\alpha$ source; black: pure source with background subtracte).

\section{Long Term Stability}

In order to check the stability of the detector in long term robust running, we used $\operatorname{Ar}(98 \%)+C H 4(2 \%)$ at $20 \mathrm{kPa}$ and $\mathrm{HV} 1 / \mathrm{HV} 2=3220 \mathrm{~V} / 950 \mathrm{~V}$ to monitoring $\alpha$ response, with a threshold of 4000 ADC for weeks running. The raw trigger rates is stable $1 \mathrm{~Hz}$ at room temperature and pressure, and the response keeps almost constant as shown in Fig. 11, where the $\alpha$ location keeps stable around 1\%. The stability of the energy resolution is a better test of that the out gassing and vacuum leakage are not interfering with the

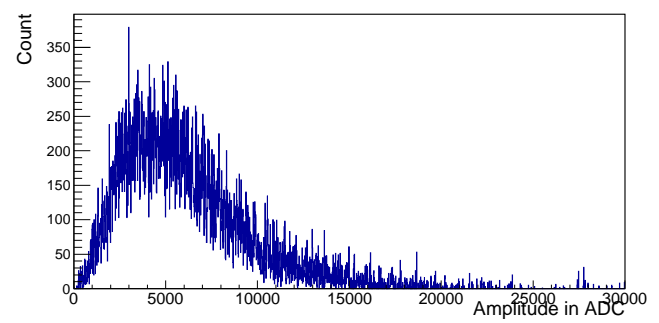

Figure 10: Amplitude spectrum after rise time cut $[20,60] \mu$ s for X-ray. 
long term stability, while it still need more calibration and monitoring for further information.
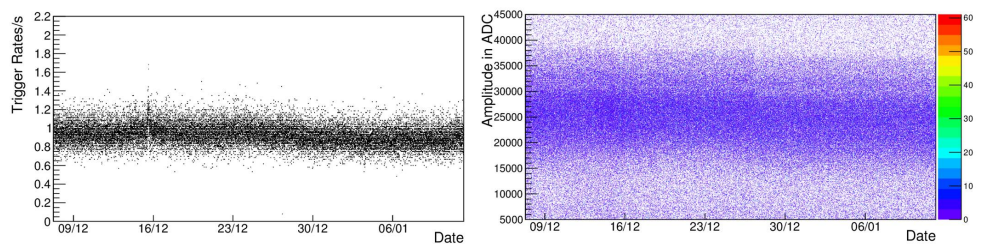

Figure 11: Long term stability monitoring: left panel is the raw trigger history; right panel is the $\alpha$ peak fluctuation.

\section{Summary}

The SPC, in principle, is capable of detecting very low energy nuclear recoils (in the few tens keV energy region) and smaller energy with lower pressure, thus being a very good candidate for rare event searches. Preliminary understanding was achieved with a SPC prototype. It can measure $\alpha$ and neutron, and show the capability of particle identification. Long term running of the detector shows that it is a good stable detector. It needs more tests and study to reach lower energy region, and better particle identification, more understanding of efficiency etc.

\section{Acknowledgement}

This research/publication is supported by the National Natural Science Foundation of China (11475205) and Tsinghua University for its grant to Charling Tao and the study.

\section{References}

[1] J D VergadosJournal of Physics: Conference Series, 2007, Vol.65(1), p.012002 (11pp)

[2] Vergados, J D ; Giomataris, Y ; Novikov, Yu N, Journal of Physics: Conference Series, 2010, Vol.259(1), p.012100 (6pp)

[3] Vergados, J D, Journal of Physics: Conference Series, 2011, Vol.309(1), p.012031 (15pp) 
[4] Bougamont, E etc., Journal of Modern Physics, Jan, 2012, Vol.3(1), p.57(7)

[5] Vergados, J D ; Moustakidis, Ch C, Journal of Physics: Conference Series, 2011, Vol.315(1), p.012028 (8pp)

[6] Vergados, J D Journal of Physics: Conference Series, 2012, Vol.384(1), p.012023 (8pp)

[7] Galan, Javier etc, Arxiv ID: 1310.4092

[8] Bougamont, E etc. Journal of Physics: Conference Series, 2011, Vol.309(1), p.012023 (8pp)

[9] Bougamont, E etc, Journal of Physics: Conference Series, 2013, Vol.460(1), p.012016 (8pp)

[10] Giomataris, I et al, Nuclear Physics B, 2006, Vol.150, pp.208-213

[11] Ali Dastgheibi-Fard et al, Physics 2014 0148-6349

[12] S Aune et al, Physics 2005 No.11 0148-6349

[13] Aune, S et al, J.Phys.Conf.Ser. 39 (2006) 281-283

[14] I.Savvidis et al, arXiv:1606.02146v1 [physics.ins-det] 7 Jun 2016

[15] Bougamont E, Dastgheibi A, Derre J, et al. Neutron spectroscopy with the Spherical Proportional Counter based on nitrogen gas $[\mathrm{J}]$. Physics, 2015, tipp2014. 